\title{
CRITICAL SUCCESS FACTORS IN MANAGING VIRTUAL TEAMS: FRAMEWORK AND RELATIONSHIPS
}

\author{
Ganesh Vaidyanathan, Indiana University South Bend, gvaidyan@iusb.edu \\ Asghar Sabbaghi, Indiana University South Bend, Sabbaghi@iusb.edu \\ Carmen Debrot, IBM Corporation, Venezuela, cdebrot@ve.ibm.com
}

\begin{abstract}
The corporate world is changing from boardroom meetings and group gatherings to a virtual team world. Mobile employees who work from home or a remote office are becoming more common. More and more companies are adopting virtual teams because they bring many advantages to organizations and their employees. However, virtual teams carry additional complexity and increase risk of unsuccessful projects in terms of reaching their goals. This paper defines virtual teams, discusses their advantages and challenges, and identifies several success factors to manage virtual teams. These factors are aggregated using a fourdimensional framework. This paper discusses factors from the structural, cognitive, relational, and technological dimensions of the framework and the relationships between them. Finally, relationships between some factors of the four dimensions and performance of virtual teams are analyzed.
\end{abstract}

Keywords: Virtual teams, success factors, structural, cognitive, relational, technical, framework

\section{INTRODUCTION}

Loughran [1] states that virtual teams are defined as groups of distributed people working together to achieve a common goal. They solve a shared problem through the use of computer-mediated communication technologies, linking them across time, space, and cultural barriers. There are different elements that can make a team virtual, such as geographical separation of team members, skewed working hours, temporary or matrix reporting structures, and multi-corporation or multiorganizational teams [2]. Townsend et al [3] defined virtual teams as groups of geographically and/or organizationally dispersed coworkers that are assembled using a combination of telecommunications and information technologies to accomplish an organizational task. Whirlpool Corporation used a virtual team composed of experts from the United States, Brazil, and Italy during a two-year project aimed at developing a chlorofluorocarbon- have different shapes and styles using various combinations of whether they belong to same or different organizations and whether they belong to same or cultures and nationalities. Virtual teams allow organizations to access the most qualified individuals for particular skills regardless of their location, enable organizations to respond quickly to increased competition, and provide greater flexibility to individuals working from home or on the road. The implementation of virtual teams is a growing trend that has become an integral part of our society. The rapid and substantial growth of information and communication technologies has allowed the fast development of virtual teams. In addition to technology, other factors that have contributed to the prevalence of virtual teams are mergers, acquisitions, downsizing, outsourcing, technology, and technical specialization [2]. At present, more and more employees are opting for tele-work alternatives. Teleworkers currently make up the fastest growing segment of the workplace [5].

In this paper, we plan to propose a framework that may be used to measure success in managing virtual teams. Such a framework is not available in the current literature. This framework may be used by managers to analyze the need for virtual teams as well as to measure success through the employment of such teams. The next section illustrates the formation of such a framework along with discussions of impacts of the framework factors on each other.

\section{LITERATURE SURVEY}

Haywood [2] has identified different advantages of virtual teams. She presented the advantages from both the managers' perspective and team members' perspective. The managers' perspectives are generally based on the factors that include access to a less expensive labor pool, reduced office space, greater utilization of employees, round-the-clock work force, greater access to technical experts, and larger pool of possible job experience. However, the perspectives of team members includes factors such as increased independence, less micromanagement, larger pool of jobs to choose from, greater flexibility, and opportunity for travel. While some of these factors may seem opposing in thought processes, it is necessary that both managers and team members 
understand how virtual teams benefit all participants, in order that the implementation of virtual teams be successful. Telecommuters show an increase of productivity between $15 \%$ and $80 \%$ [6]. This advantage is not limited to work-at-home workers; also employees working on projects headquartered at geographically remote locations have improved their productivity because they have a more structured and less-interrupt-driven communication style. There are studies suggesting that when virtual teams are given sufficient time to develop strong intra-group relationships and adapt to communications medium, they may communicate as effectively as face-to-face groups [7].

Virtual teams can be implemented to reduce susceptibility to environmental disaster, such as what the Oracle Corporation's customer support division has done. IBM implemented a hoteling for its marketing and services personnel that reduced by $60 \%$ the real estate costs per location. Commuters produce millions of gallons of exhaust fumes per year. Studies in the United Kingdom showed that it is possible to eliminate 1.2 tons of carbon dioxide by taking one commuter off the road. Xerox sent their sales and support personnel to work at customer sites in one location, due to the necessity of employees to spend more time at a client's site because of an increase in product complexity.

However, there are different perspectives of the challenges of implementing virtual teams in these two groups. According to Haywood [2], $70 \%$ of the time the concerns of the managers have to do with control, including monitoring performance, and training and mentoring of new employees. The second area of concern is communication. In addition, they are also worried about team building, cultural issues, and cost and complexity of technology, process, and workflow. The areas of most concerns among team members are communication and support. Team members are worried about being excluded from key meetings and decisions. Technical support is an overwhelming concern for non-technical team members. In addition, they are worried about recognition, inclusion vs. isolation and management resistance. Also, they may be concerned that all promotions go to team members working at the same site as the manager.

\section{A FRAMEWORK TO ANALYZE SUCCESS FACTOR TO MANAGE VIRTUAL TEAMS}

In face-to-face environments, team members, attending the same meetings, working in the same physical environment, tend to share commonly held information, and experiencing similar organizational culture. They all contribute to a common understanding of team goals. However, in virtual team settings contextual knowledge may be held uniquely and tends to be unevenly distributed among team members. For example, failure to share the contextual knowledge in virtual team environments may cause misunderstandings or misinterpretation of a remote team member's behavior, and negatively affect the performance of the team.

Different authors have identified several success factors to manage virtual teams and such factors can be aggregated in different dimensions. Vaidyanathan [8] developed a four-dimensional framework of distributed project knowledge management. These dimensions are identified in figure 1. The structural dimension refers to the pattern of relationships between the project team members $[9,10]$. This dimension consists of the following factors that include ties, configuration, or the pattern of linkages among team members, stability, informality, and

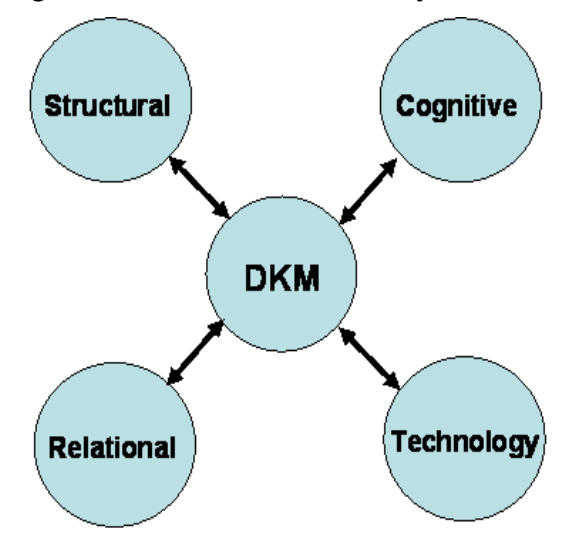

Figure 1. Four dimensions of Distributed Project Knowledge Management management of project teams.

The cognitive dimension involves resources that provide shared meaning and understanding between project team members. This dimension contains factors that include shared goals, shared culture, and learning. Shared goals include collective goals, aspiration, and a shared vision for project team members. If project team members are culturally diverse, the different cultures need to be understood and accommodated. At the same time, the team members in order to be effective should be trained on social mores and aware of local human resources practices. The project team should have an integrated learning culture that includes education, training, and mentoring. 
The relational dimension is centered on the role of the direct ties between team members. This dimension factors focuses on shared responsibility for outcome, trust and collaboration among distributed project teams. Trust in a firm focuses on the role of direct ties between actors and the relational, as opposed to structural, outcomes of interactions [10]. Trust is a key element in the willingness of team members to share knowledge. In particular, collective trust can be a crucial element of vitual team well functioning. Collective trust is defined as a shared psychological state in a team that is characterized by an acceptance of vulnerability based on expectations of of intensions and behaviors of others within the team [11]. Collaboration is defined as the degree to which team members actively help one another in their work [12]. Trust plays an important role in successful collaboration.

The technical dimension refers to IT tools, support, and maintenance since the virtual team relies on technology-mediated communications rather than face-to-face interactions to accomplish their task. IT tools include system designs, technology acceptance and adoption, compatibility, ontology, and security. IT facilitates collection, storage, and exchange distributed project knowledge, and foster all modes of knowledge creation [13]. Traditionally, there has been much focus on synchronous rather than asynchronous technologies. Asynchronous technologies, which include email and discussion forums, are common in the business world [14]. Furthermore, asynchronous technologies offer certain advantages for groups exchanging information and may allow group members to concentrate on message content. For example, individuals can take time to reflect on the message they receive and to carefully consider their responses.

Relational and Structural Dimensions

Strong ties promote trust and collaboration. As trust develops over time, opportunities for knowledge transfer between members should increase [10]. Configuration of a team should encourage collaboration as configuration influences the flexibility and ease of communication and knowledge exchange between team members. A decentralization of authority to members such as the development of lateral ties improves communication and collaboration [10]. Strong ties encourage trust. Moreover, the concentration of decision-making authority inevitably reduces creative solutions, whereas the dispersion of power facilitates spontaneity and experimentation, and the freedom of expression which are the lifeblood of knowledge creation [13]. In addition, leadership should promote trust and collaboration.

The stability of a virtual team increases trust and collaboration. In particular, collective trust is an essential element of any virtual team in order to function effectively. There is more difficulty in collaboration if there is a high personnel turnover. Highly unstable network may limit opportunities for the creation of social capital, because when an actor leaves the network, ties disappear [10]. Using these arguments, we propose

\section{Preposition 1:}

Relational and structural dimensions are positively related.

\section{Relational and Technical Dimensions}

Duarte and Snyder [15] consider that actions of a team leader and team members that impact trust fall into three categories to include performance and competence, integrity, and concern for the well-being of others. They state that all three factors should exist in order for a virtual team to have a high level of trust. For each category, Duarte and Snyder [15] specify many factors that are included in Table 1. free refrigerator [4]. Virtual teams can

\begin{tabular}{|c|c|}
\hline Category & Factors \\
\hline Performance and competence & $\begin{array}{l}\text { Developing and displaying competence } \\
\text { Following through on commitments and showing results }\end{array}$ \\
\hline Integrity & $\begin{array}{l}\text { Ensuring that your actions are consistent with your words } \\
\text { Standing up for your convictions; displaying integrity } \\
\text { Standing behind the team and its members } \\
\text { Communicating and keeping everyone informed about } \\
\text { progress } \\
\text { Showing both sides of an issue }\end{array}$ \\
\hline $\begin{array}{l}\text { Concern for the well-being of } \\
\text { others }\end{array}$ & $\begin{array}{l}\text { Helping team members with transitions } \\
\text { Being aware of your impact on others } \\
\text { Integrating team needs with other teams, department, and } \\
\text { organizational needs }\end{array}$ \\
\hline
\end{tabular}


In order to select the right technology for a global virtual team, it is necessary to consider the nature of the task and the context where it occurs. Context has an impact on the reliability of the communication technology on which members depend [16]. In addition, local habits are developed in a determined context and can have big variations across team members. Gibson and Cohen [16] identify aspects to consider choosing the right technology that include infrastructure, differences in local power, telephone or cable infrastructure, culture and language, accessibility of information, time zone gap, team size, technology maturity, and task complexity.

Technology can send subtle messages about which members of the team are considered high performer or not. The communication between high performers tends to be through one-on-one electronic messages. Thus, being left out of one-on-one communication patterns could indicate that a team member is perceived as less competent than others [15]. Regarding the second factor, the integrity of team processes and decision making can be facilitated through technology. For example, groupware with anonymity features allows sharing opinions and ideas, especially when there is a disagreement or when only a few members of the team do not share an idea. Using technology, members can express their opinion without fear of recrimination. In addition, electronic distribution lists make it easy to get the same information to everyone in a timely manner [15]. In relation to the third factor, the fact that virtual teams operate in an isolated environment can generate less need for social posturing than in traditional teams. This situation can produce a tendency to display less concern. One study showed that computer-mediated groups communicate more negative messages than face-to-face groups do [15]. To avoid this situation, members of virtual teams should avoid waiting for face-to-face meetings or at least tele-conference to express criticism in order to avoid misunderstanding.

In summary, we propose that

Proposition 2a:

Use of technology can have a positive impact on performance and competence as well as integrity factors of trust

\section{Proposition 2b:}

Use of technology has a negative impact on concern for the well-being of others.

\section{Proposition 2c:}

Use of technology has a positive impact on team collaboration.

\section{CONCLUSION AND FUTURE RESEARCH}

Virtual teams offer an opportunity to organizations enabling them to handle a more demanding and competitive work environment and, at the same time, present many potential challenges. Different factors of the dimensions explained above have a diverse impact on the performance of the virtual team. In particular, the relationship between trust and virtual team performance has been analyzed by various authors. Also, the relationship between conflict management and virtual team performance has been studied extensively. Regarding the relationship between trust and virtual team performance, different studies have shown contradictory results. A number of studies show the positive effects that trust has on performance. However, more recent studies have failed to find a positive relationship between trust and performance [17].

There is a distinction between efficiency and effectiveness to explain the lack of the relationship between trust and virtual team performance. Trust does not necessarily augment the quality of the task performance. Jarvenpaa, Shaw, and Staples [17] found no relationship between trust and task performance. Moreover, trust can have unpleasant consequences including that high levels of trust may not always be justified because of the risk that others will take advantage of the situation.

Montoya-Weiss, Massey, and Song [18] explore the relationship between conflict management behavior and virtual team performance. They distinguish five conflict handling modes to conflict management in an organization: avoidance, accommodation, competition, collaboration, and compromise. They found that avoidance conflict management behavior has a significantly negative effect on performance. However, contrary to the authors' expectation, accommodation had no significant effect on performance. This situation is different from traditional non-virtual teams where past research has found a negative relationship between accommodation and performance. The explanation of the authors is that because of the use of asynchronous communication environment, it is possible that, no matter how much an individual may express accommodation, the team does not experience it [18]. In addition, these authors found positive relationships between collaboration conflict management of behavior and performance. Montoya-Weiss, Massey, and Song [18] have established a negative 
relationship between compromised conflict management and performance.

Virtual teams have being adopted by many corporations. They have many advantages as well as challenges. These challenges can be aggregated in structural, cognitive, relational, and technological dimensions. We are investigating the relationships between relational and structural dimensions. We believe that strong ties promote trust and collaboration, team configuration affects collaboration, and team stability increases trust and collaboration. We are also investigating the relationship between relational and technical dimensions. We believe that technology factors have an impact in building trust in virtual teams. In addition, technology has a role in development virtual teams. Finally, we feel that performance of virtual teams is impacted by different factors of these four dimensions. In particular, trust and conflict management has an impact in the performance of virtual teams.

The implications of the findings from this study can be classified into three categories - contribution to literature, insights about various dimensions affecting virtual teams, and insights about the impacts of these factors on virtual team performance. From a practical standpoint, we present a framework to examine virtual team. This framework offers a promising avenue for future research. Future studies can use this framework to examine the performance of virtual teams and measure various factors on how they impact the performance.

\section{REFERENCES}

1. Loughran, J. (2005). Working Together Virtually: The Care and Feeding of Global Virtual Teams. Retrieved February 15, 2005 from http://www.thoughtlink.com/files/htmldocs/TLI-ICCRTS00.htm

2. Haywood, M. (1998). Managing Virtual Teams: Practical Techniques for High-Technology Project Managers. Boston: Artech House.

3. Townsend, A. M., DeMarie, S. M., \& Hendrickson, A. R. (1998). Virtual teams: technology and the workplace of the future. Academy of Management Executive, 12, 17-29.

4. Geber, B. (1995). Virtual teams. Training, 32, 36-40.

5. Johnson, P., Heimann, V., \& O’Neil, K. (2001). The wonderland of virtual teams. Journal of Workplace Learning 13(1), 24-30.
6. Lauzon, E. (2008). Featured Article: Beating telework challenges pays off in energy savings. Retrieved on May 12, 2010 from http://www.nortel.com/corporate/pressroom/feat ure_article/collateral/feature_teleworking.pdf.

7. Chidambaram, L. (1996). Relational development in computer-supported groups. MIS Quarterly, 20(2), 143- 163.

8. Vaidyanathan, G. (2005). Networked Knowledge Management Dimensions in Distributed Projects. International Journal of e-Collaboration, 2(4), 19-36.

9. Nahapiet, J., \& Ghoshal, S. (2001). Social capital, intellectual capital, and the organizational advantage. Academy of Management Review, 23(2), 242-266.

10. Inkpen, A.C., \& Tsang, E.W.K. (2005). Social capital, networks, and knowledge transfer. Academy of Management Review, 30(1), 146166.

11. Rousseau, D. M., Sitkin, S. B., Burt, R. S., \& Camerer, C. (1998). Not so different after all: a cross-discipline view of trust. Academy of Management Review, 22, 393-404.

12. Hurley, R. \& Hult, T. (1998). Innovation, market orientation, and organization learning: An integration and empirical examination. Journal of Marketing, 62(3), 42-54.

13. Lee, H., \& Choi, B. (2003). Knowledge management enablers, processes, and organizational performance: An integrative view and empirical examination. Journal of Management Information Systems, 20(1), 179228.

14. Kinney, S. T., \& Panko, R. R. (1996). Project teams: profiles and member perceptionsimplications for group support system research and products. Proceedings of the Twenty-Ninth Hawaii International Conference on System Sciences, Kihei, Maui, 128-137.

15. Duarte, D., \& Snyder, N. (1999). Mastering Virtual Teams. San Francisco: Jossey-Bass Publishers.

16. Gibson, C., \& Cohen, S. (2003). Virtual Teams that Work. San Francisco: Jossey-Bass Publishers.

17. Jarvenpaa, S., Shaw, T., \& Staples, D. (2004). Toward contextualized theories of trust: the role of trust in global virtual teams. Information System Research, 15(3), 250

18. Montoya-Weiss, M., Massey, A., \& Song, M. (2001). Getting it together: temporal coordination and conflict ement in global virtual teams. Academy of Management Journal, 44(6), 1251-1262. 